\title{
Effect of Inertial Measurement Unit on Creating High-Definition 3D Map for Autonomous Vehicle
}

\author{
Nang Xuan Ho ${ }^{1,2 *}$, Anh Son Le $e^{1,2}$ \\ ${ }^{I}$ Phenikaa Research and Technology Institute, Phenikaa Group, Hanoi, Vietnam \\ ${ }^{2}$ Faculty of Vehicle and Energy Engineering, Phenikaa University, Hanoi, Vietnam \\ *Email: nang.hoxuan@phenikaa-uni.edu.vn
}

\begin{abstract}
One of the most famous technologies for the autonomous vehicle was using a scan matching algorithm, in which a high-definition 3D map created by the LIDAR sensor plays a significantly important role in localizing and path planning. Within this manuscript, a novel way of finding the effect from the Inertial Measurement Unit (IMU) on creating a high-definition 3D map from the LIDAR sensor was investigated. The collection data system was first ever created and collected in Vietnam. The results show that the normal distributions transform shows very good performance for creating the HD 3D map with have IMU sensor. On the other hand, without IMU the accuracy and the robustness of the creating map were reduced especially in the nonflat area. This manuscript will start the evolution of preparation for autonomous vehicles in Vietnam as well as contribute to the autonomous vehicle research society in the world.
\end{abstract}

Keywords: HD map, autonomous vehicle, IMU.

\section{Introduction}

According to the World Health Organization, a traffic accident is a critical problem for the world, it takes approximately 1.35 million people to die each year and it is still increasing [1]. One of the ideas is an autonomous vehicle, which can significantly reduce traffic accidents in the future.

According to several researchers, to be ready for an autonomous vehicle, we have to prepare the facility included drive-by-wire vehicles, maps, and sensors (Fig.1). After that, the sensing data, perception, planning, control, localization should be completed to make a full system of the autonomous vehicle [2-8]. Here we only focus on the creation of a high definition (HD) 3D map.

HD map for AV is not a new technology in the world, but in Vietnam, there is still no HD map database or HD map companies. The application of 3D LIDAR for making point clouds is limited. There are only some companies and groups that use photogrammetry techniques to make 3D point clouds, mainly for geology and architecture conservation purposes. To realize AV in Vietnam shortly, it is necessary to develop both HD map databases and tools/devices.

There are two kinds of techniques for creating a point cloud map [9]: 1/ Using camera (image) to

ISSN 2734-9373

https://doi.org/10.51316/jst.150.ssad.2021.31.1.5

Received: January 01, 2021; accepted: May 12, 2021 extract the point cloud by adding the global position system (GPS) information (Fig.2) and 2/ using the LIDAR (Fig.3).

The point cloud is a set of points $(x, y, z)$ in a space created with many purposes such as use in shaping, architecture, and especially applied to the survey of traffic works or using used to convert to vector maps for future autonomous vehicles. In the case of using the camera for creating a point cloud map, the series of pictures will be taken with the global positioning system (GPS) information. Each picture will be processed to be a set of the vector point, and then by comparing the nearest set of vector points of the next picture, the combination will combine two sets to become one and creating a new point cloud map.

In the case of using LIDAR, all of the information from LIDAR will be collected. The scan matching algorithm will be applied for comparing with the previous scan and then combine them together.

Furthermore, the HD map is not only using for vehicle navigation but also using for the vehicle to understand the surrounding environment such as lane, traffic signal location, road type, ... by adding the information to the HD map (Fig.4).As recommended by the previous researcher [10], we have selected the second technology by using LIDAR 3D to create the HD 3D map and also investigate the effect of IMU on creating an HD map in Vietnam, which does not exist before. 
JST: Smart Systems and Devices

Volume 31, Issue 1, May 2021, 033-040

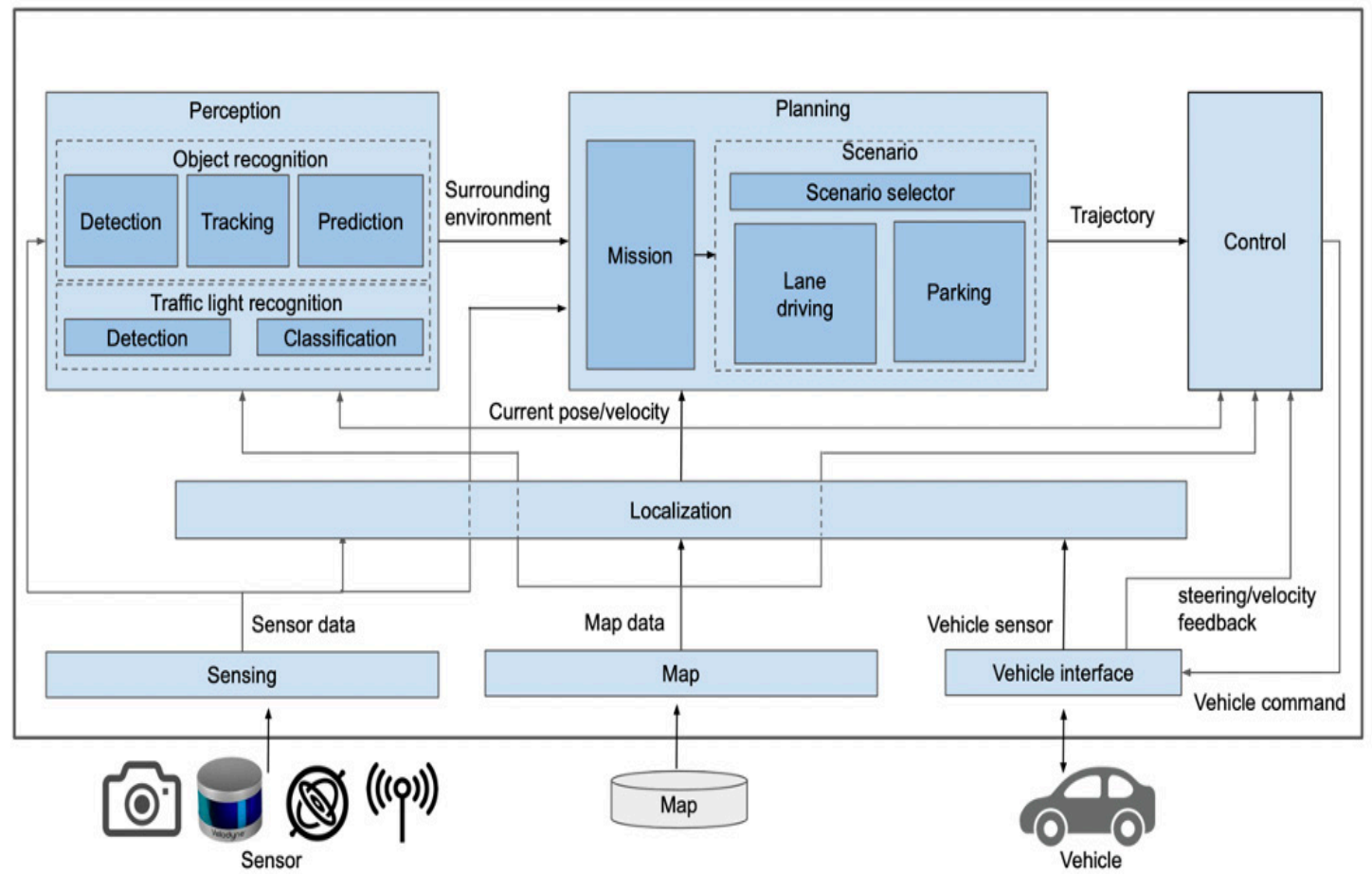

Fig.1. A typical autonomous vehicle system overview

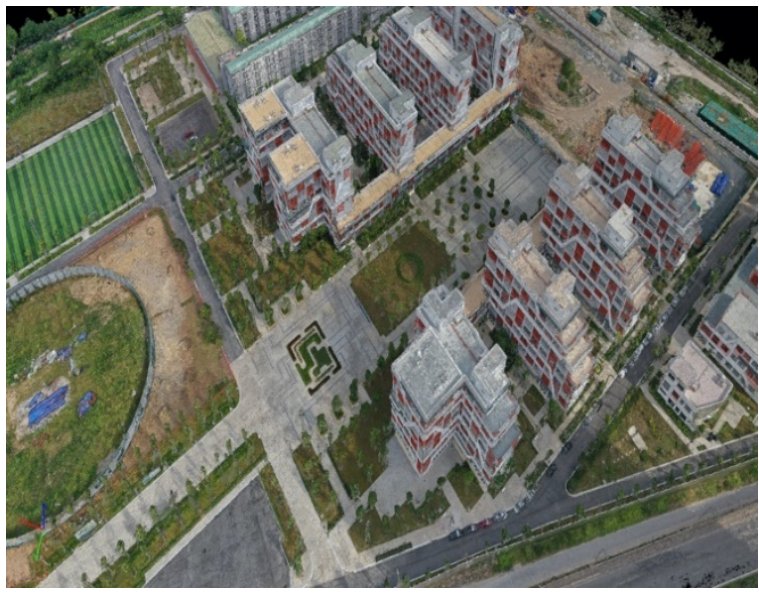

Fig. 2. Point Cloud map created by image

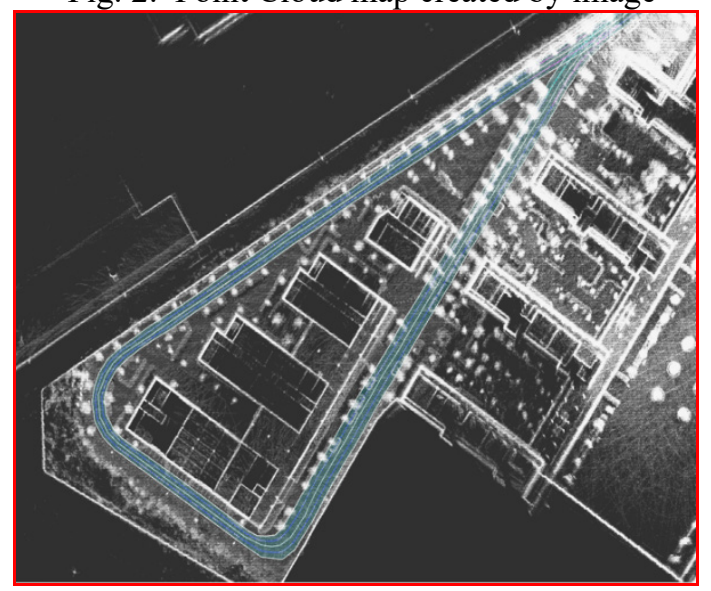

Fig. 4. Adding information for HD map

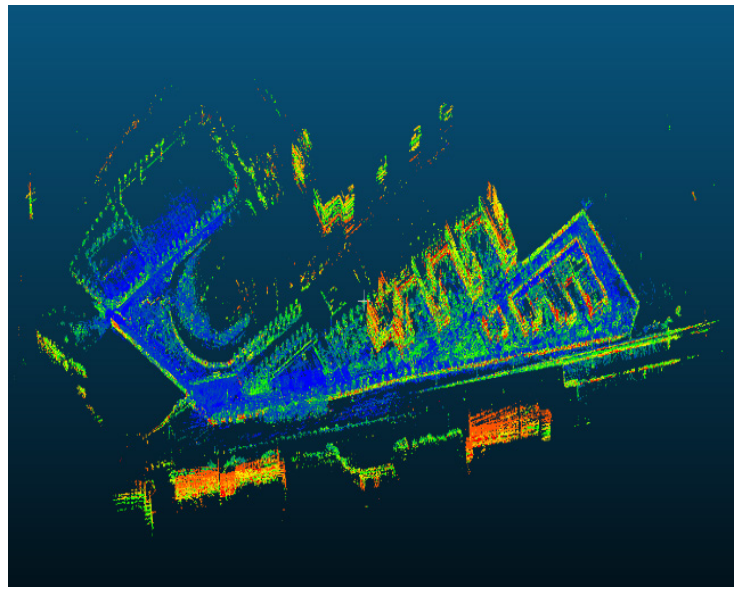

Fig. 3. Point Cloud map created by LIDAR

\section{Materials and Methods}

\subsection{The Mathematic Calculation for Creating an HD $3 D$ Map}

For creating the HD 3D map, Biber et al. introduced the Normal Distributions Transform (NDT) scan matching method by using point cloud and divided it into a uniform grid [11]. Each voxel (cell) uses the mean and distribution of the sub-point cloud assigned to it. After that, this algorithm was developed by Takeuchi et al to enhance the scanmatching by adding the dual resolutions of NDT [12]. In this manuscript, we applied the same technique with Takeuchi, the equation shows below: 


$$
\begin{aligned}
& p_{k}=\frac{1}{M_{k}} \sum_{i=1}^{M_{k}} x_{k i} \\
& \sum_{k}=\frac{1}{M_{k}} \sum_{i=1}^{M_{k}}\left(x_{k i}-p_{k}\right)\left(x_{k i}-p_{k}\right)^{T}
\end{aligned}
$$

while $x_{i}=\left(x_{i}, y_{i}, z_{i}\right)^{T}$ with $i=1: M$;

Denote $R$ as the rotation matrix and $t^{\prime}$ as the translation vector, the $x_{i}$ can be calculated by:

$$
x_{i}^{\prime}=R x_{i}+t^{\prime}
$$

The pose translation and rotation parameters to be estimated are

$$
\begin{gathered}
t=\left(t_{x}, t_{y}, t_{z}, t_{\text {roll }}, t_{\text {pitch }}, t_{\text {yaw }}\right) \\
E(X, t)=\sum_{i}^{N} \exp \frac{-\left(x_{i}^{\prime}-p_{i}\right)^{T} \sum_{i}^{-1}\left(x_{i}^{\prime}-p_{i}\right)}{2}
\end{gathered}
$$
aligned.

$E(X, t)$ represents the matching or the well-

A high value of $E(X, t)$ means both the input cloud and the reference map are well aligned. Newton's nonlinear function optimization is utilized to find $t$ such that $E(X, t)$ is maximized. Therefore, we minimize the function $f(t)=-E(X, t)$.

The parameter $t$ will be updated using:

$$
t_{\text {new }}=t-H^{-1} g
$$

where $g$ and $H$ are the partial differentials and second-order partial differential of the optimizing function $f$.

Basically, without IMU, the t parameter will be estimated through equation (5), which is not considered about the $z$-axis (the normal global positioning system doesn't have $z$-axis information). Furthermore, with the high frequency from IMU, the $t$ parameter will be updated more often. The processing data was shown in Fig.5.

\subsection{Data Acquisition System and Test Area}

- Data acquisition system

For data logging (Fig.6), a hard-ware system was created by:1/an SUV vehicle; 2/ The LIDAR sensor that has a measurement range up to $100 \mathrm{~m}$ with a range accuracy of $\pm 3 \mathrm{~cm}$ and a vertical field of view from $+15^{\circ}$ to $-15^{\circ}$. The rotation rate can be adjusted from 5 to $20 \mathrm{~Hz}$; 3/ Real-time kinematic (RTK): The GNSS system namely Piksi-Multi evaluation kit with high accuracy; and 4/ IMU namely Xsens Mti-G-710 with gyroscope range +/- 450 degrees/s with bias stability 10 degrees/hour.

All of the signals from IMU, LIDAR, RTK will be processed through a high specification computer by using Robot Operation System (ROS). All of the mathematic for scan matching was done in the ROS.

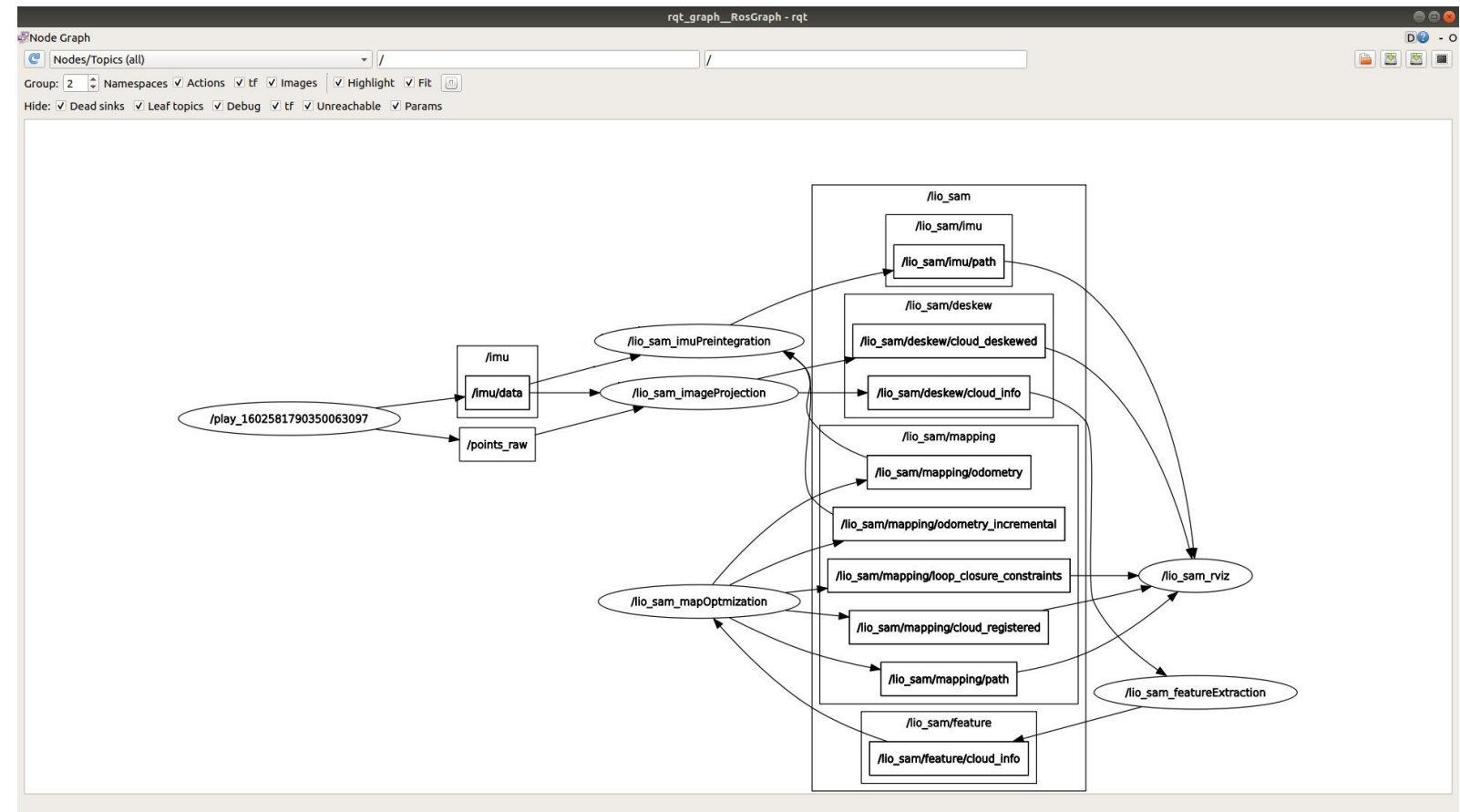

Fig. 5. Data process on ROS 


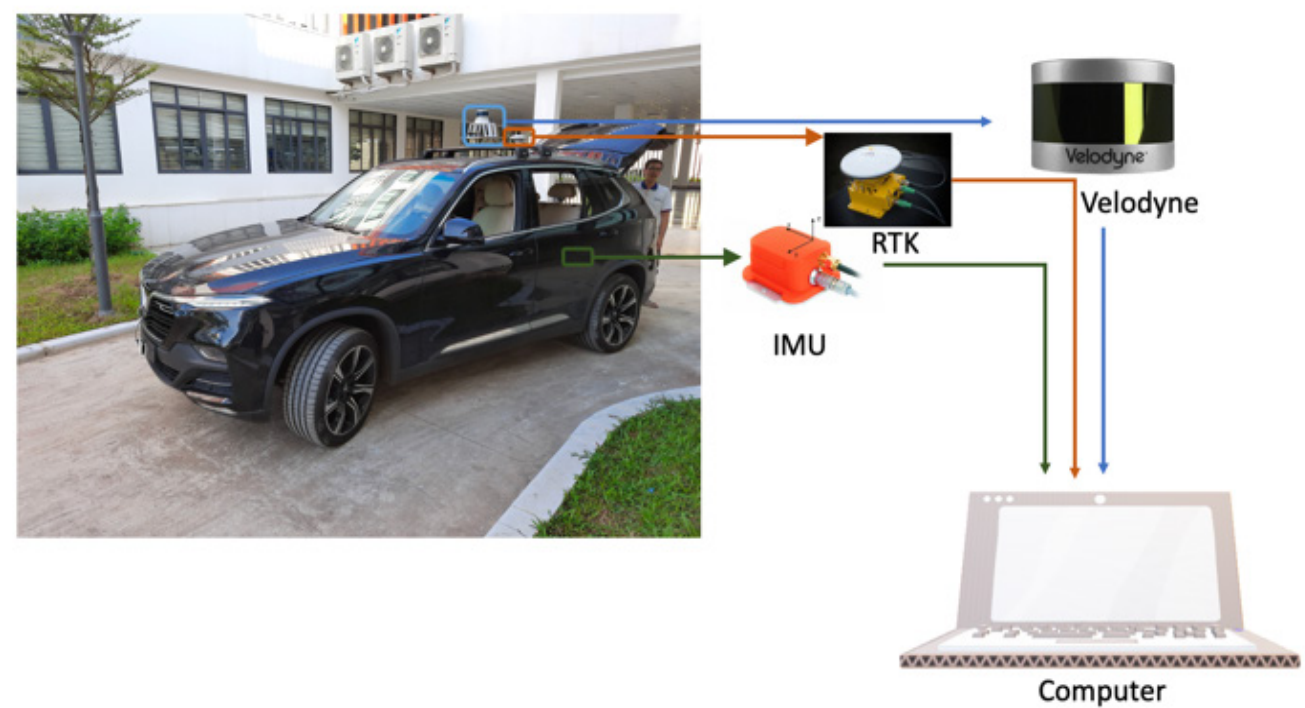

Fig. 6. Data acquisition system.

- $\quad$ Testing area and strategy

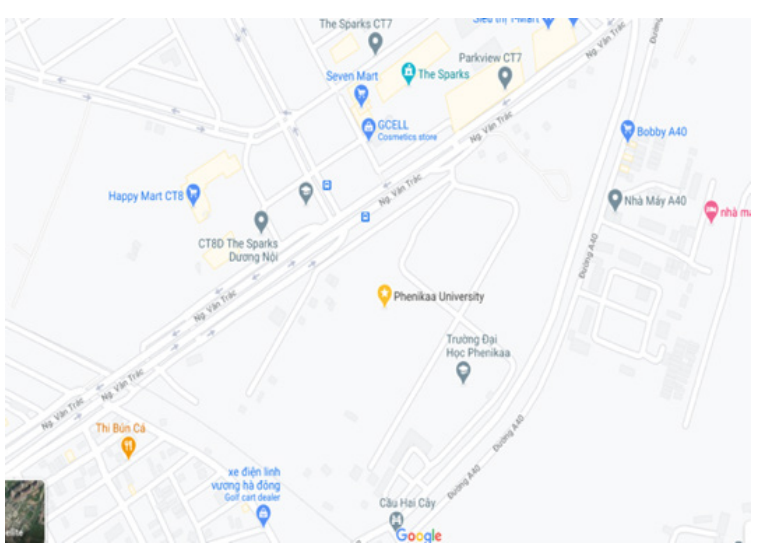

Fig. 7. Testing area.
In this study, we collected data at Phenikaa University (Fig.7), which is located in Hanoi, Vietnam with two scenarios: 1/ Inside the campus of Phenikaa University; and 2/ The campus and the road in front of the campus with the overpass.

\section{Results}

\subsection{Data Collection}

The data collected show very good performance with clear data (Fig.8), especially for the LIDAR signal. All of the data will be input to the ROS and process the NDT algorithm for scan matching and then create the HD map with two cases: with and without IMU by removing the IMU topic.

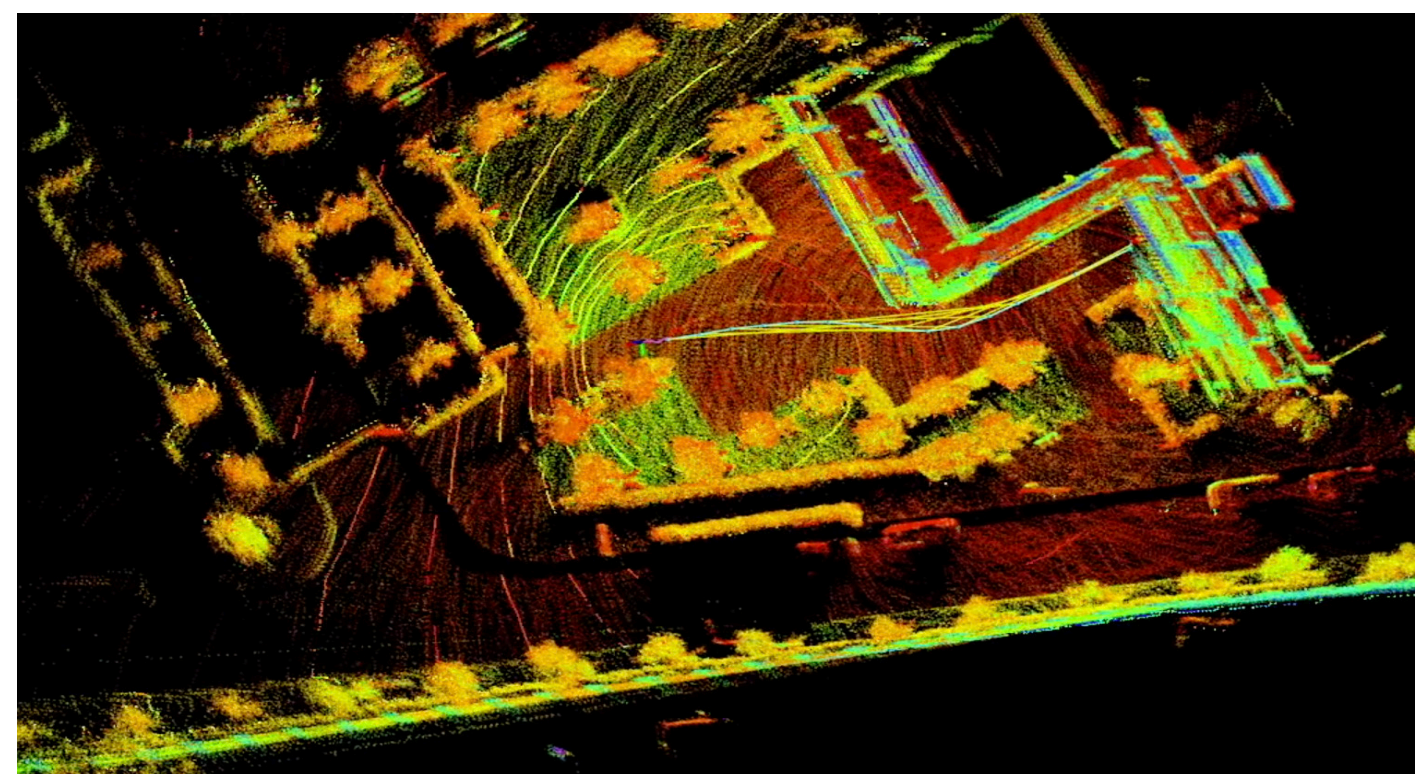

Fig. 8. Creating the map. 


\subsection{Map creation inside the Phenikaa University}

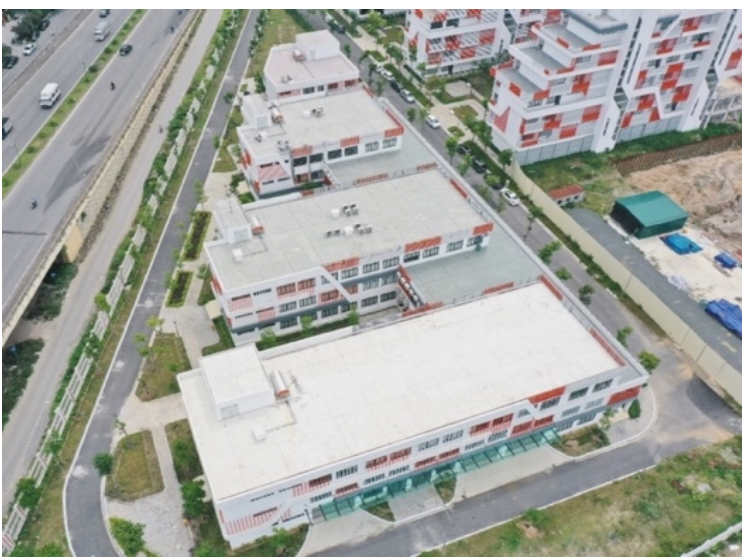

Fig. 9. A view of Phenikaa University.

Phenikaa University is located in a flat area, south-west of Hanoi. The University just rebuilding all of the facilities so the road condition is good

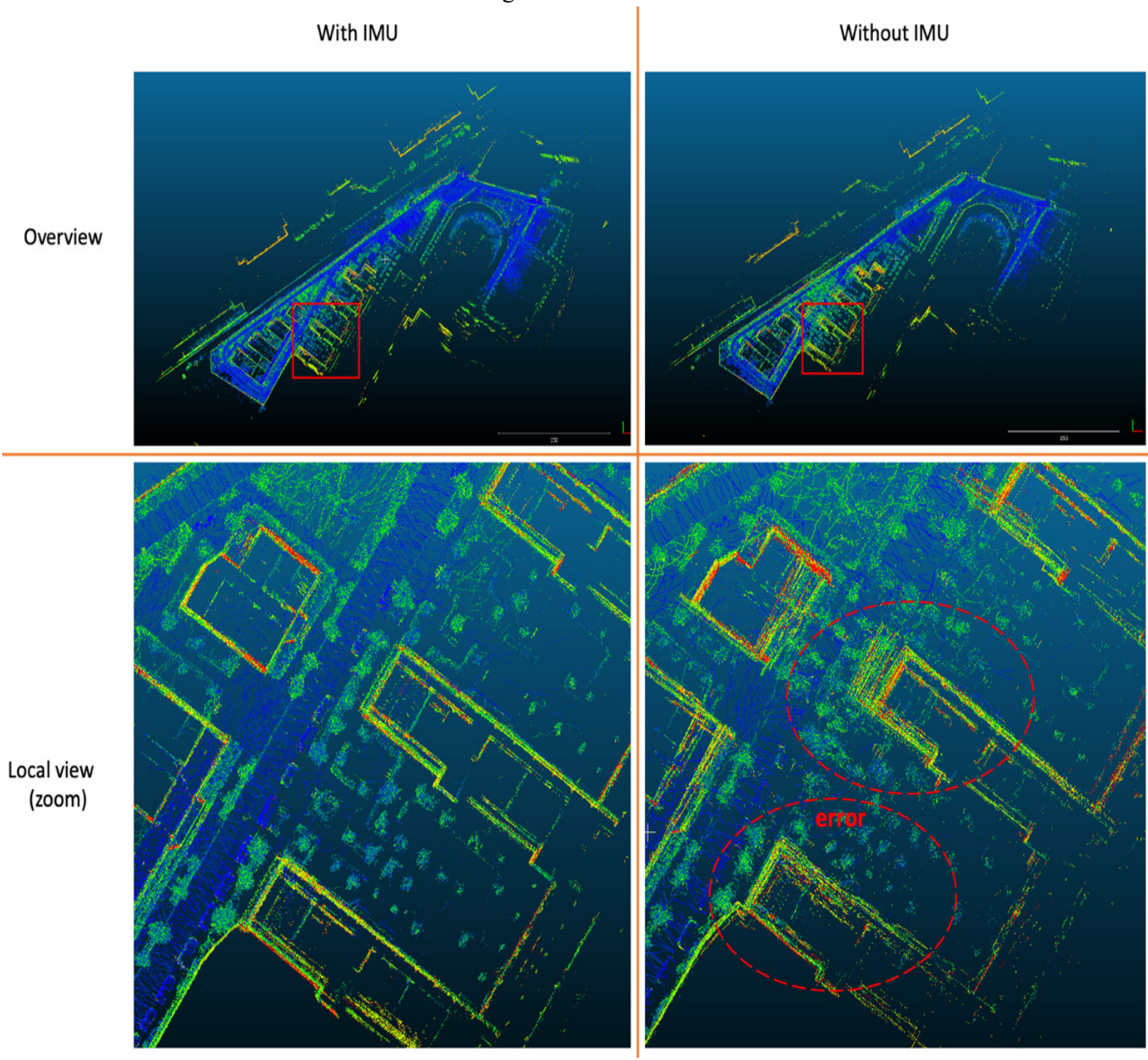

Fig. 10. Map creation inside the campus.

(Fig.9). There is not much vibration when the vehicle moves with low speed for mapping creation.

The NDT shows very good performance with a high density of point cloud in both case with and without IMU. However, while zoom some parts in detail, the map creation is shown some error while matching the scan point in case of without IMU (Fig.10).

In Fig.10, the overview of the HD map shows almost the same due to the flat area, and the vehicle moves slowly. However, at some corners, there is some vibration exit and it may make the scan matching work not so well. In this case of local closed-loop street mapping, both methods with and without using IMU data were able to deliver a usable point cloud of the area, as shown in Fig.10. However, as we zoomed up to the detail of each point cloud data, matching errors were found on the building's walls in the case of not using IMU data.

Without IMU 


\subsection{Map Creation Outside the Phenikaa University}

In this case, a long straight main road with no closed-loop was chosen. There is an overpass in one ending of the testing area, which offers a largealtitude change in point cloud data. Moreover, there are not many marks on the overpass, which makes it more challenging for the 3D matching without using IMU. Fig. 11 shows very clearly in detail the effect of IMU, as we zoomed up to the detail of each point cloud data, matching errors were found on the side view, overpass in the case of not using IMU data. Furthermore, a clear and shaped point cloud was delivered in the case of using IMU data during point cloud generation. On the contrary, in the case of not using IMU data, only unusable data was formed. In this data, although the top-view of the main road part seems to be similar to the 2D-map shape, there were still matching errors on the building's wall, which is similar to the first strategy case when closely looking into detail of the point cloud. Besides, the side view of the point cloud shows that the main road is bent during the point matching process, which makes the height of the road changed incorrectly when comparing with terrestrial data. On the other hand, the NDT-matching method also failed to generate the detail of the overpass part of the main road where the logging vehicle's altitude is suddenly changed. In conclusion, IMU is found to be an essential secondary sensor that provides stable performance for a mapping system.

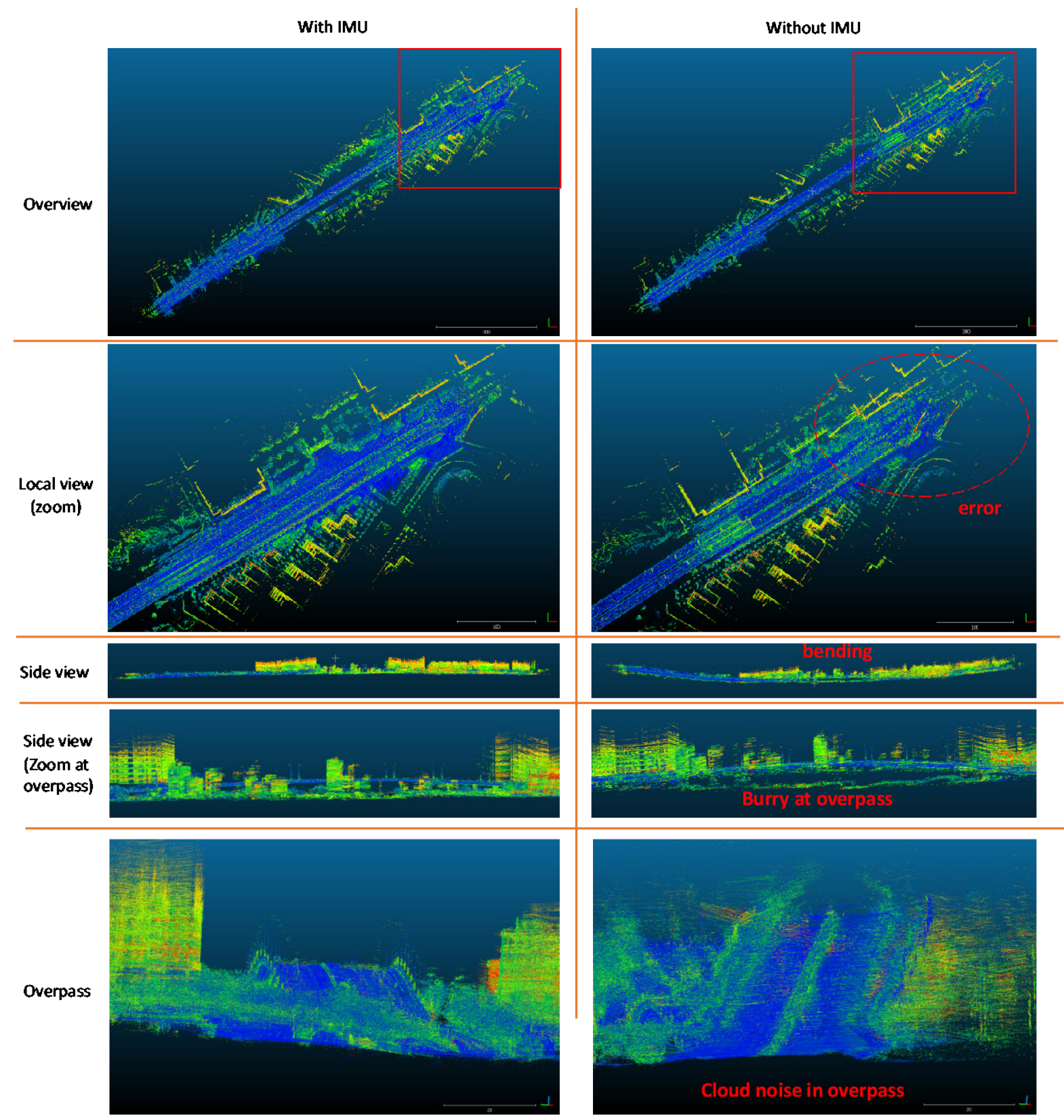

Fig. 11. Map creation inside the campus. 


\section{Conclusion and Outlook}

The revolution for transportation namely autonomous vehicles can make a significant change such as reducing accidents and congestion will be real soon by overpassing many technical challenges [13]. One of the key for autonomous driving in the city is HD maps, which is used for accurate localization [14]. Here, a novel creating the HD maps as well as the effect of the IMU sensor on the making HD map in Vietnam was shown. Basically, IMU, which is an electronic device that measures the orientation based on accelerometers, plays a very important role in navigation such as using with global position system to calculate EKF [15]-[17]. At this time, the result also indicated that IMU has a strong influence on creating HD maps. By collecting the information of $x, y, z$ axis, the IMU can help the scan matching algorithm remove the noise as well as enhance the accuracy by giving exactly the altitude, which is necessary for the vehicle while running in a non-flat area.

To make autonomous level 5 happened shortly, we still have to overcome a lot of challenges not only inside the vehicle but also the facility preparation. However, with the revolution of the sensors, the computer, the algorithm, we believed that the autonomous vehicle will be a key point for changing the society in near future.

The map plays a very important role in the autonomous vehicle especially for path planning and localization. In this manuscript, the effect of IMU on the creating map has been shown. The results indicated that without IMU, the map creation may have a lot of effects such as cloud noise, incorrect potion, burry. It may help other researchers have a deep looking into the design system and move forward to make autonomous vehicles happened.

\section{Acknowledgments}

This work was partly supported by Phenikaa University.

\section{References}

[1] Road traffic injuries, 2020 [Online]. Avaiable: https://www.who.int/news-room/factsheets/detail/road-traffic-

injuries\#: :text=Approximately 1.35 million people die,result of road traffic crashes.\&text=More than half of all,pedestrians $\% 2 \mathrm{C}$ cyclists $\% 2 \mathrm{C}$ and motorcyclists.

[2] Á. Arcos-García, J. A. Álvarez-García, and L. M. Soria-Morillo, Evaluation of deep neural networks for traffic sign detection systems, Neurocomputing, vol. 316, pp. 332-344, 2018, https://doi.org/10.1016/j.neucom.2018.08.009.

[3] H. Sobreira et al., Map-Matching Algorithms for Robot Self-Localization: A Comparison Between Perfect Match, Iterative Closest Point and Normal Distributions Transform, J. Intell. Robot. Syst.
Theory Appl., vol. 93, no. 3-4, pp. 533-546, 2019 , https://doi.org/10.1007/s10846-017-0765-5

[4] L. Caltagirone, M. Bellone, L. Svensson, and M. Wahde, LIDAR-camera fusion for road detection using fully convolutional neural networks, Rob. Auton. Syst., vol. 111, pp. 125-131, 2019, https://doi.org/10.1016/j.robot.2018.11.002.

[5] L. Wang, Y. Zhang, and J. Wang, Map-Based Localization Method for Autonomous Vehicles Using 3D-LIDAR., IFAC-PapersOnLine, vol. 50, no. 1, pp. 276-281, 2017, https://doi.org/10.1016/j.ifacol.2017.08.046.

[6] L. Wang, Y. Zhang, and J. Wang, Map-Based Localization Method for Autonomous Vehicles Using 3D-LIDAR, IFAC-PapersOnLine, vol. 50, no. 1, pp. 276-281, 2017,

https://doi.org/10.1016/j.ifacol.2017.08.046

[7] S. D. Pendleton et al., Perception, planning, control, and coordination for autonomous vehicles, Machines, vol. 5 , no. 1 , pp. $1-54,2017$, https://doi.org/10.3390/machines5010006

[8] E. Javanmardi, Y. Gu, M. Javanmardi, and S. Kamijo, Autonomous vehicle self-localization based on abstract map and multi-channel LIDAR in urban area, IATSS Res., vol. 43, no. 1, pp. 1-13, 2019, https://doi.org/10.1016/j.iatssr.2018.05.001.

[9] R. Liu, J. Wang, and B. Zhang, High Definition Map for Automated Driving: Overview and Analysis, J. Navig., vol. 73, no. 2, pp. 324-341, 2020, https://doi.org/10.1017/S0373463319000638

[10] A. Carballo et al., Characterization of Multiple 3D LIDARs for Localization and Mapping using Normal Distributions Transform, no. April, 2020, [Online]. Available: http://arxiv.org/abs/2004.01374.

[11] P. Biber, The Normal Distributions Transform: A New Approach to Laser Scan Matching, IEEE Int. Conf. Intell. Robot. Syst., vol. 3, no. November 2003, pp. 2743-2748, 2003, https://doi.org/10.1109/IROS.2003.1249285

[12] E. Takeuchi and T. Tsubouchi, A 3-D scan matching using improved 3-D normal distributions transform for mobile robotic mapping, in IEEE International Conference on Intelligent Robots and Systems, 2006, https://doi.org/10.1109/IROS.2006.282246

[13] M. Martínez-Díaz, F. Soriguera, and I. Pérez, Autonomous driving: A bird's eye view, IET Intell. Transp. Syst., vol. 13, no. 4, pp. 563-579, 2019. https://doi.org/10.1049/iet-its.2018.5061

[14] H. G. Seif and X. Hu, Autonomous Driving in the iCity-HD Maps as a Key Challenge of the Automotive Industry, Engineering, vol. 2, no. 2, pp. 159-162, 2016. https://doi.org/10.1016/J.ENG.2016.02.010

[15] J. Kim and S. Lee, A vehicular positioning with GPS/IMU using adaptive control of filter noise covariance, ICT Express, vol. 2, no. 1, pp. 41-46, 2016

https://doi.org/10.1016/j.icte.2016.03.001 
[16] A. Ndjeng Ndjeng, D. Gruyer, S. Glaser, and A. Lambert, Low cost IMU-Odometer-GPS ego localization for unusual maneuvers, Inf. Fusion, vol. 12, no. 4, pp. 264-274, 2011.

https://doi.org/10.1016/j.inffus.2010.06.006

[17] L. Wei, C. Cappelle, Y. Ruichek, and F. Zann,
Intelligent vehicle localization in urban environments using EKF-based visual odometry and GPS fusion, IFAC Proc. Vol., vol. 44, no. 1 PART 1, pp. 1377613781, 2011,

https://doi.org/10.3182/20110828-6-IT-1002.01965 